\title{
Chronological Progress of Calcified Lesion in the Iliofemoral Vein in a Patient with Chronic Renal Failure
}

Koki Eto*, Yoji Kubo, Reiko Kemmochi and Mitsuaki Matsumoto

Department of Cardiovascular Surgery, Cardiovascular Center, Tsuyama Chuo Hospital, Tsuyama, Japan

${ }^{*}$ Corresponding author: Koki Eto, Department of Cardiovascular Surgery, Cardiovascular Center, Tsuyama Chuo Hospital, Tsuyama city, Okayama, Japan, Tel: 81868218111; Fax: 81868218201; Email: etouk.tchshinge@tch.or.jp

Rec date: Feb 04, 2015; Acc date: Feb 16, 2015; Pub date: Feb 18, 2015

Copyright: () 2015 Eto K, et al. This is an open-access article distributed under the terms of the Creative Commons Attribution License, which permits unrestricted use, distribution, and reproduction in any medium, provided the original author and source are credited.

\begin{abstract}
We report the case of a 64-year-old man refered to our department for edema and tiredness in legs, who had been followed up for chronic renal failure and secondary hyperparathyroidism. He had no history of medical treatment for deep venous thrombosis, however, computed tomography (CT) revealed extensive calcification in veins of the lower half of the body. Since peripheral venous calcification is a rare disease and advances slowly, imaging the chronological progress of calcification is difficult. This case suggests the usefulness of CT for diagnosing and treating peripheral venous calcification.
\end{abstract}

Keywords: Peripheral venous calcification; Computed tomography; Chronic renal failure

\section{Abbreviations \\ CT: Computed Tomography}

\section{Case Report}

A 64-year-old Japanese male was examined because of edema and tiredness in legs. He had been followed up for 14 years due to chronic renal failure, maintained by hemodialysis, and secondary hyperparathyroidism, treated with oral calcium and sevelamer hydrochloride. His recent laboratory data, including calcium (8.4 $\mathrm{mg} / \mathrm{dL}$ ) and blood coagulation factor levels, were within almost normal limits, with the exception of hemoglobin $(9.8 \mathrm{~g} / \mathrm{dL})$, phosphorus $(7.0 \mathrm{mg} / \mathrm{dL})$ and intact parathormone $(114 \mathrm{pg} / \mathrm{ml})$. His ankle-brachial pressure index showed normal range. Although the patient had no history of medical treatment for deep venous thrombosis, computed tomography (CT) revealed extensive calcification in veins of the lower half of the body (Figure 1). These were especially severe in the iliofemoral veins and had significantly worsened over the past 6 years (Figure 2). He did not have ectopic calcification other than the vesseles. Surgical repair, such as femoral venous drainage to inferior vena cava, was considered if his symptoms were to worsen.

Although it has been reported that hepatocirrhosis can occasionally lead to venous calcification in portal vein, the mechanisms behind venous calcification are poorly understood. In these patients, calcification was typically located at most in the superior mesenteric vein. Previous reports have revealed that long-term indwelling central venous catheters can increase the risk of thrombus formation and rarely become the cause to organize central venous calcification following such a thrombus [1]. In the present case, however, the patient did not have any history of catheterization, and his venous calcification continued to peripheral veins below his knee [2].
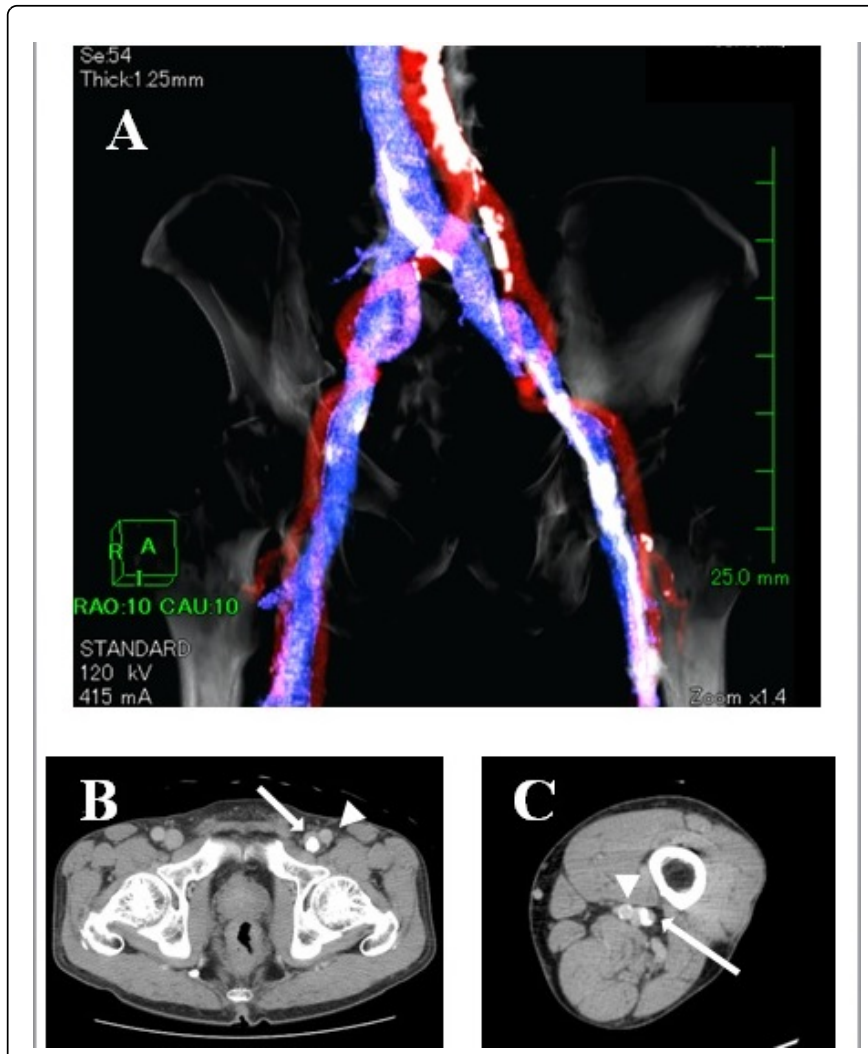

Figure 1: (A) Three-dimensional computed tomography. Highdensity areas (white), confined in vein (blue) and around artery (red), indicate calcifications. (B) Axial images showing intravenous calcification in left common femoral vein, (C) continuous calcification in left popliteeal vein. Arrows indicate the vein, and arrowheads indicate the artery. 

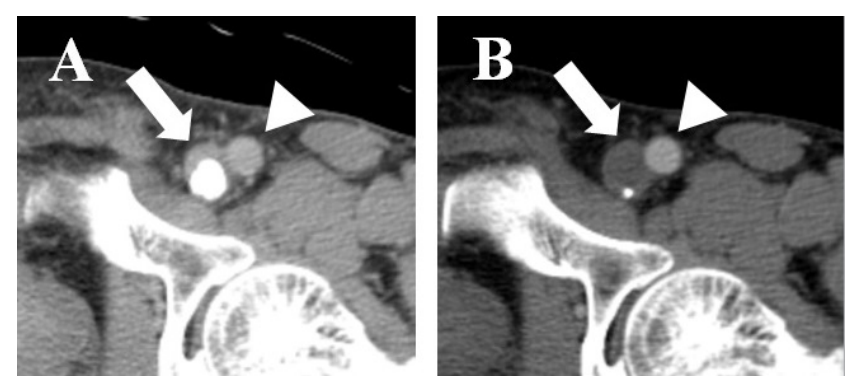

Figure 2: Axial images from enhanced computed tomography. Current venous calcification in common femoral vein $(12.3 \mathrm{~mm}$, A), increased significantly compared to that 6 years ago $(2.4 \mathrm{~mm}$, B). Arrows indicate the vein, and arrowheads indicate the artery.

The patients who are treated by chronic renal failure occasionally lead to arterial calcification associated with secondary hyperparathyroidism, whereas venous calcification, especially occurred in peripheral vein in lower limbs, is rare. These are the reason why we can still not reveal the cause of venous calcification in our patient. In patients of atherosclerosis obliterans, intermittent claudication and psychroesthesia in lower limbs, for example, begin to appear because of ischemia. However, venous calcification advances slowly and patients are generally asymptomatic, and thus it is difficult to diagnose and image the chronological progress of venous calcification in peripheral veins. CT is thought to be the most sensitive examination technique to detect calcification, followed by ultrasonography and radiography. In our case, the patient underwent
CT by chance and had not exhibited any symptoms in the lower limbs for the previous 6 years [3]. To our knowledge, this is the first report of clearly imaged progressed venous calcification in the lower limbs with using three-dimensional CT. We believe that this technique may be useful for diagnosing and safely treating vascular diseases, including venous stenosis and occlusion, as the present case.

\section{Authors' Contributions}

KE wrote the manuscript and collected references. MM, YK and RK were primarily involved in the clinical and scientific discussion of the case and commented to this manuscript. All authors read and approved the final manuscript.

\section{Acknowledgements}

We thank cardiologists, clinical engineers, radiological technologists and nurses at Cardiovascular Center, Tsuyama Chuo Hospital, without whom this work would not have been possible.

\section{References}

1. Verma V, Cronin DC, Dachman AH (2001) Portal and mesenteric venous calcification in patients with advanced cirrhosis. AJR Am J Roentgenol 176: 489-492.

2. Hughes DB, Ullery BW, Spigland N (2006) Formation of a calcified "cast" in a long-term indrewelling central venous catheter: a case report. J Pediatr Surg 41: 1927-1929.

3. Ayuso C, Luburich P, Vilana R, Bru C, Bruix J (1992) Calcification in the portal venous system: comparison of plain films, sonography, and CT. AJR Am J Roentgenol 159: 321-323. 